\title{
Assessment of Knowledge on management of Pulmonary Tuberculosis under RNTCP among graduating Interns and Postgraduate students in RIMS Imphal.
}

\author{
Dr Bandi K Sangma ${ }^{1}$, Dr Bhavya Shivalingaiah ${ }^{2}$, Prof W Asoka Singh ${ }^{3}$, \\ Dr Lalfakzuala Renthlei ${ }^{4}$ Dr Rosswel Sangma ${ }^{5}$ \\ ${ }^{I}$ Junior Resident, Department of Respiratory Medicine, RIMS, Imphal, Manipur, India. \\ ${ }^{2}$ Senior resident, department of respiratory Medicine, KIMS, Karnataka Bangalore, India. \\ ${ }^{3}$ Professor, Department of Respiratory Medicine, RIMS,Imphal, Manipur, India. \\ ${ }^{4}$ Junior Resident, Department of Respiratory Medicine, RIMS, Imphal, Manipur, India. \\ ${ }_{5}^{5}$ Junior Resident, Department of social \& preventive Medicine, RIMS, Imphal, Manipur, India.
}

\begin{abstract}
RNTCP was started in 1997 and has covered the entire nation in March 2006. At this time it also became to be known as RNTCP II, which started to initiate services to TB/HIV, MDR-TB and to extend RNTCP to the private sector. With RNTCP both diagnosis and treatment of TB are free. There is also atleast in theory, no waiting period for patients seeking treatment and TB drugs.

Materials and Methods: This observational, cross-sectional study was conducted in 2016 among a total of 133 graduating interns and PG students. Study tool was in english, pre-designed, pre-tested, self administered, semi constructed questionaire, to collect information relating to transmission, diagnosis and treatment of pulmonary tuberculosis.The data collected was tabulated, analysed and interpreted by proper statistical methods.

Results: $99.2 \%$ of the participants answered droplet as the main mode of transmission for pulmonary tuberculosis. $75 \%$ answered cough for more than two weeks as the important symptom. $90.2 \%$ answered sputum microscopy as the investigation of choice for diagnosing TB under RNTCP. 66.7\% answered sputum microscopy as the choice for follow up. 78.8\% answered two sputum samples were needed for diagnosis. More than $60 \%$ answered correctly regarding categories, phases, duration of treatment and first line ATT drugs. More than $65 \%$ answered correctly regarding the management of tuberculosis under special conditions like infancy, children less than 6 years and pregnant women. $45 \%$ of the participants could correctly define new TB patients, relapse case, default, MDR-TB XDR-TB and TB suspect. $51.5 \%$ answered that a family member could be a DOT provider.78\% of the participants correctly defined supervised treatment. $30 \%$ of the participants correctly answered the diagnostic tests banned in India. $68 \%$ of the participants linked RNTCP to NACO. More than $95 \%$ could directly define the acronym of DOTS and RNTCP.Only $12.1 \%$ had any kind of TB training.

Conclusion: The study revealed the lack of knowledge of TB signs and symptoms, diagnosis and treatment. Poor level of knowledge can prevent medical practitioners from suspecting TB and its inadequate management can lead to treatment failure and drug resistance.
\end{abstract}

Keywords: Interns, Knowledge, pulmonary tuberculosis, RNTCP.

\section{Introduction}

Millions are believed to have died of Tuberculosis, while the real cause was "Lack of Awareness". [1] Unlike smallpox and plaque whose reign has been short-lived, TB has perpetually gone on unabated, torturing and killing ceaselessly. RNTCP was started in 1997 and has covered the entire nation in March 2006. With RNTCP both diagnosis and treatment of TB are free. There is also at least in theory, no waiting period for patients seeking treatment and TB drugs.

According to World Health Organization (WHO) Global Tuberculosis Report, in 2013, an estimated 9.0 million people developed tuberculosis and 1.5 million died from the disease. India is the highest TB burden country with World Health Organization (WHO) statistics for 2011 giving an estimated incidence figure of 2.3 million cases of TB for India. The estimated TB prevalence figure for 2011 is given as 3.1 million. It is estimated that $40 \%$ of the Indian population is infected with TB bacteria, the vast majority of whom have latent rather than active TB.[2] A subtle but fundamental shift has thus taken place. Onus of cure rests not any more with the patient but with the health care system and which, empowered with personal information about the patient's whereabouts, retains the ultimate initiative. Delay in TB case finding is a common problem affecting TB prevention and control throughout the world. Globally, studies have shown that individual's delay in TB diagnosis is due to low knowledge, low risk perceptions and other social and demographic factors such as age, education, sex and economic status. 


\section{Materials And Methods}

A cross sectional study was conducted in tertiary care hospital Regional Institute of Medical Sciences Imphal, Manipur, India. Study tool was a pre-tested self-administered questionnaire, which were distributed to the students in the hostel/hospital and collected after about 15 minutes. The study was intended to cover all Interns and PGT in RIMS Imphal. Those who refused to participate and who could not be contacted after 3 consecutive visits were excluded from the study. Prior approval was obtained from the Institutional Ethics Committee, RIMS Imphal before starting the study. Informed consent was taken before distributing the questionnaire and confidentiality of the participants were maintained. Study duration was from September 2016 to November 2016.

There were 26 questions for evaluating the knowledge regarding tuberculosis management under RNTCP. The maximum attainable score was 26 and minimum 0 (zero). Categorization was done as: Inadequate knowledge (score below the median) and adequate knowledge (score above median). Data collected were checked for consistency and completeness and analyzed using SPSS version 21 (IBM). Descriptive statistics like mean, percentage and proportion were used. Chi square test was used to assess the association between selected variables and a P-value $<0.05$ was taken as statistically significant

\section{Results}

\section{Response rate:}

Response of interns was $93.75 \%$ (80) and that for PGTs was $41.42 \%(140)$

"Table 1: Distribution of Sociodemographic profile and other variables" $(\mathrm{n}=133)$

\begin{tabular}{|l|l|l|}
\hline \multicolumn{1}{|c|}{ Characteristics } & Number & Percentage \\
\hline Gender & & \\
\hline Male & 75 & $56.4 \%$ \\
\hline Female & 58 & $43.6 \%$ \\
\hline Level of study & & \\
\hline Intern & 75 & $56.4 \%$ \\
\hline PGT & 58 & $43.6 \%$ \\
\hline RNTCP training & & \\
\hline Yes & 16 & $12 \%$ \\
\hline No & 117 & $88 \%$ \\
\hline
\end{tabular}

During the period of study a total of 133 doctors (interns and post graduates) participated in the study. Of the total number of participants $56.4 \%(\mathrm{n}=75)$ were males and $43.6 \%(\mathrm{n}=58)$ were females. Interns comprised $56.4 \%(\mathrm{n}=75)$ and PGTs comprised $43.6 \%(\mathrm{n}=58)$. However only $12 \%(\mathrm{n}=16)$ had received any kind of training in RNTCP during their period of study in RIMS, the remaining students $88 \%(\mathrm{n}=117)$ did not have any kind of training.

"Table 2: Proportion of correct answers to knowledge questions"

\begin{tabular}{|l|l|l|}
\hline Knowledge item & No. of correct responses & Percentage \\
\hline Chief mode of transmission of PTB & 132 & $99.2 \%$ \\
\hline Most important symptom of PTB & 100 & $75.2 \%$ \\
\hline Investigation of choice for diagnosing TB under RNTCP & 120 & $90.2 \%$ \\
\hline Investigation of choice for follow up of cases with TB & 89 & $66.7 \%$ \\
\hline Number of sputum samples needed for diagnosis & 105 & $78.9 \%$ \\
\hline Most important timing for sputum sample collection & 61 & $45.5 \%$ \\
\hline No. of categories under RNTCP & 84 & $63.2 \%$ \\
\hline No. of phases in which treatment is given & 124 & $93.2 \%$ \\
\hline Duration of treatment for each category & 116 & $87.2 \%$ \\
\hline First line anti-TB drugs used in DOTS & 110 & $82.6 \%$ \\
\hline No of times DOTS therapy is given in a week & 104 & $78.8 \%$ \\
\hline Age group to which DOTS caters & 88 & $66.2 \%$ \\
\hline Prophylaxis of a child<6yrs of mother with active PTB & 91 & $68.2 \%$ \\
\hline Management of pregnant mother with PTB & 96 & $72.0 \%$ \\
\hline Definition of new TB patients & 61 & $45.9 \%$ \\
\hline Definition of relapse & 123 & $92.5 \%$ \\
\hline Definition of default & 114 & $85.7 \%$ \\
\hline Definition of MDR TB & 112 & $84.2 \%$ \\
\hline Definition of XDR TB & 74 & $55.6 \%$ \\
\hline Family member as DOT provider & 47 & $34.8 \%$ \\
\hline Definition of supervised treatment & 104 & $78.2 \%$ \\
\hline Definition of TB suspect & 85 & $62.9 \%$ \\
\hline No of persons one smear positive pt can infect annually & 57 & $43.2 \%$ \\
\hline Newer diagnostic techniques banned in India & 40 & $30.1 \%$ \\
\hline Reading of montoux test & 87 & $65.2 \%$ \\
\hline RNTCP linked to other national programme & 91 & $68.4 \%$ \\
\hline
\end{tabular}


For the question on the mode of transmission of pulmonary TB as droplet infection, it was correctly answered by $132(99.2 \%)$. For the question regarding the most important symptom of pulmonary tuberculosis, $100(75.2 \%)$ correctly answered cough for more than two weeks as the most important symptom. Investigation of choice for diagnosing tuberculosis with sputum microscopy was correctly answered by 120(90.2\%) and $105(78.9 \%)$ correctly answered two as the total number of sputum samples to be collected. $89(66.7 \%)$ correctly answered sputum microscopy as investigation of choice for follow up of cases of pulmonary tuberculosis. However only $61(45.5 \%)$ correctly answered the morning sample as the most important timing for sputum collection. For the question regarding the number of categories in RNTCP, only 84 (64.2\%) could correctly answer two categories. 124(93.2\%) could correctly answer the number of phases in which DOTS is given in RNTCP and $116(87.2 \%)$ correctly answered the question of duration of treatment of cat-I as 6 months and cat-II as 8 months. $110(82.6 \%)$ of the respondents correctly answered the names of the first line ATT as rifampicin, isoniazid, pyrazinamide, ethambutol and streptomycin. For the question regarding the number of times DOTS therapy is given in a week 104(78.8\%) correctly answered it to be thrice in a week and $88(68.2 \%)$ of the respondents correctly answered that DOTS caters to all age groups. However only 91(68.2\%) correctly answered isoniazid prophylaxis for a child less than 6 years with a mother of active pulmonary tuberculosis. Regarding the management of a pregnant female on ATT, 96(72.0\%) correctly answered that except for streptomycin all the first line ATT drugs can be given. The definition of new TB patients, relapse, default, MDR-TB, XDR TB and TB suspect was correctly defined by 61(45.9\%), 123(92.5\%), 112(84.2\%), 74(55.6\%) and $85(62.9 \%)$ of the respondents respectively. Only $47(34.8 \%)$ of respondents correctly answered that family member cannot be a DOT provider. For the question regarding the number of persons one smear positive patient can infect annually, 57(43.2\%) correctly answered 10 to 15 persons in a year. Serological tests for TB being banned in India was correctly answered by only $40(30.1 \%)$ of the respondents. $87(65.2 \%)$ of the respondents correctly answered that tuberculin reaction is read by measuring the induration and 91(68.4\%) correctly answered the question on RNTCP being linked to NACO (national AIDS control organization)

“Table 3: Relationship between knowledge adequacy of respondents and different characteristics. ” $(\mathrm{n}=133)$

\begin{tabular}{|l|l|l|l|}
\hline \multirow{2}{*}{ Characteristics } & Knowledge & \multirow{2}{*}{ P value } \\
\cline { 2 - 3 } & Adequate n(\%) & Inadequate $\mathrm{n}(\%)$ & \\
\hline Male & $44(58.7)$ & $31(41.3)$ & 0.691 \\
\hline Female & $36(62.1)$ & $22(37.9)$ & \\
\hline Postgraduate & $46(79.3)$ & $12(20.7)$ & 0.000 \\
\hline Interns & $34(45.3)$ & $41(54.7)$ & \\
\hline RNTCP training & $12(75)$ & $4(25)$ & 0.196 \\
\hline No training & $68(58.1)$ & $49(49.9)$ & \\
\hline
\end{tabular}

Mean score obtained was 18 (with a standard deviation of 3.14). Median score attained was 18 (Inter quartile range of $16-20$ ). Minimum score obtained was 8 and maximum score obtained was 25 . Table 3 shows the relationship between knowledge adequacy of respondents and different characteristics $(n=133)$. Between the two groups, postgraduate students $46(79.3 \%$ ) had adequate knowledge as compared to interns where only $34(45.3 \%)$ had adequate knowledge, which was statistically significant ( $\mathrm{p}$ value $<0.05)$. 44(58.7\%) of the male respondents had adequate knowledge as compared to female respondents, where only $36(62.1 \%)$ had adequate knowledge. However it was not statistically significant. Among the group with RNTCP training, only $12(58.1 \%)$ had adequate knowledge regarding pulmonary TB management under RNTCP.

\section{Discussion}

Similar kinds of studies have been carried out in a number of institutions throughout India. Most of them also reveal a low or inadequate knowledge for the management of pulmonary tuberculosis according to the revised national tuberculosis program (RNTCP).

Bogam R.R et al carried out a study in Pune city, which revealed inadequacies in the knowledge of tuberculosis amongst postgraduate students.[3] In the study most of the students had low knowledge regarding diagnosis and treatment services under RNTCP. $52.77 \%$ could correctly respond to the number of sputum samples required for diagnosis, while only $69.44 \%$ and $50 \%$ of the respondents could correctly define MDR and a new case of tuberculosis respectively. Only $47.22 \%$ could correctly answer that a family member cannot be a DOT provider for treatment of tuberculosis while $55.55 \%$ correctly answered the treatment duration for cat-I TB patient. In our study the results were similar, with $34.8 \%$ of the respondents correctly answering that a family member cannot be DOT provider and only $45.9 \%$ correctly defining a new case of TB. The respondents fared better with more than $75 \%$ correctly answering the number of sputum samples required, definition of MDR and the duration of Cat-I treatment.

Basu Mis et al carried out a study, which reflected inadequate and incomplete knowledge about TB according to Revised National Tuberculosis Program guidelines among interns of tertiary teaching hospitals of 
West Bengal in 2012.[4] In the study only 65\% of the respondents had the correct knowledge about the mode of transmission and common symptoms of TB. Less then $50 \%$ of the interns knew about the drugs used in different categories, its duration and proper monitoring of patients according to RNTCP. Our study also shows that only $45.3 \%$ of interns have adequate knowledge regarding management of TB under RNTCP.

Patle R A et al in the study entitled Knowledge about Tuberculosis and Multi-Drug Resistant Tuberculosis among Interns in Shri Vasantrao Naik Government Medical College, Yavatmal, Maharashtra, India, showed that $80 \%$ in interns had knowledge of tuberculosis, clinical features of TB, diagnosis and management whereas knowledge on causes and diagnostic tests of MDR-TB was present in less than $40 \%$ of the interns.[5] The results were as comparable to our study, with more than $80 \%$ of the respondents correctly answering the questions on causes, clinical features, diagnosis and management.

Balamurugan S. S et al carried out a similar study among the aspiring doctors in a tertiary medical college hospital, Salem, Tamil Nadu, India.[6] In the study $92 \%$ of the respondents correctly knew about the mode of transmission in pulmonary TB. Less than $80 \%$ had proper knowledge regarding diagnosis and treatment services of pulmonary TB. The results were similar to our study with $99 \%$ of the respondents correctly answering the question on chief mode of transmission of pulmonary TB and $80 \%$ answering correctly to the questions on diagnosis and treatment services.

Nalabothu S.K et al carried out a study of awareness about resistant tuberculosis among medical professionals where only $42.8 \%$ and $25.7 \%$ of the respondents could correctly define MDR and XDR TB respectively.[7] In our study $84.2 \%$ of the respondents could correctly define MDR and $55.6 \%$ of the respondents correctly defined XDR TB.

Mehta $\mathrm{D}$ et al carried out a study in a tertiary care teaching hospital in which postgraduate medical trainees and interns were surveyed.[8] The number of sputum specimens required for diagnosis under RNTCP was responded correctly by only $57 \%$ and correct categorization of tuberculosis patients was done by only $56 \%$ of the respondents. In our study $78.9 \%$ of the respondents could correctly answer the number of sputum samples required for diagnosis and $63.2 \%$ correctly answered the categorization of patients in RNTCP.

Charkazi A.R carried out study among medical interns in Northern Islamic Republic of Iran. Where a majority of interns (85.0\%) correctly identified TB as a droplet infection.[9] Only 17.5\% considered sputum smears for acid-fast bacilli (AFB) as the best test for diagnosis of pulmonary TB. Only a few participants (7.5\%) were able to identify categories of TB treatment and duration of consumption of drugs in two categories. Four fifths $(80.0 \%)$ were unable to recommended which anti-TB drugs should be used in each category. Almost all of the respondents in our study (99\%) correctly answered the chief mode for transmission for pulmonary tuberculosis and more than 70\% could correctly answer the first line anti-TB drugs used in DOTS, duration of each category, number of times the drugs are given in a week and the phases in which DOTS therapy is given.

Jumbo $\mathrm{J}$ et al in a study amongst final year medical students and doctors in Nigeria where medical students constituted $66(63.5 \%)$ of the subjects compared to 38 (36.6\%) medical doctors.[10] Overall, $47.3 \%$ of respondents had good understanding of DOTS based on their answers to the questions asked in the questionnaire. In the study medical doctors had better understanding of DOTS compared to medical students and the difference was statistically difference $(\mathrm{p}<0.026)$. However, there was no significant difference between understanding of DOTS and work experience. In our study $79.3 \%$ of the postgraduate trainees had adequate knowledge as compared to medical interns $(45.3 \%)$, which was statistically significant $(\mathrm{p}<0.000)$.

The study has revealed the inadequate knowledge of interns towards the management of pulmonary tuberculosis under RNTCP. Postgraduate students faired better than the interns, which may be because of more exposure to patients and decision making for management of TB in their day-to-day training programe. Lack of knowledge on proper management can lead to treatment failure and multi drug resistance, which is even more difficult to treat, associated with adverse drug reactions, low compliance and also an economic burden to the country.

\section{Limitations}

Some of the respondents did not return the questionnaire on the same day and more than half of the postgraduate students did not participate in the study. Practices and attitudes of the respondents were not evaluated.

\section{Conclusion}

Although the importance of research and clinical trials of TB is unquestionable, health education of the masses including the medical personnel by training and health programs to dispel the myths and stigma surrounding the disease and its treatment cannot be overemphasized in India's progress toward controlling TB"the captain of all men of death".[11] 


\section{Acknowledgements}

We thank all the medical students who participated in the study, our department faculty for their guidance and the department of social and preventive medicine for their insight and recommendations for development of the questionnaire.

\section{References}

[1]. Kakar R. TBfreeworld. Available at; http://www.tbfreeworld.org. Accessed on July 12, 2015.

[2]. WHO. Global tuberculosis report 2014 by WHO. Available at; http://www. Who.int/tb/publications/globalreport/en/. Accessed on July 12, 2015.

[3]. Bogam RR and Sagare SM. Knowledge of tuberculosis and its management practices amongst postgraduate medical students in Pune city. Natl J Community Med 2011;2(1):52-59.

[4]. Basu M, Das P. Assessment of knowledge regarding tuberculosis in the context of revised national tuberculosis control program among budding doctors. Chron Young Sci 2014;5:59-64.

[5]. Patle RA, Khakse GM. Knowledge about Tuberculosis and Multi-Drug Resistant Tuberculosis among Interns. Natl J Community Med 2014; 5(1): 51-53.

[6]. Balamurugan SS, Swaminathan and Rao S.The Awareness about Tuberculosis and DOTS among the Aspiring Doctors in a Tertiary Medical College Hospital, Salem, Tamil Nadu. Natl J R Community Med 2013;2(1): 079-148.

[7]. Sandeep KN and Surendra MK. A Study of Awareness about Resistant Tuberculosis among Medical Professionals. Sch. J. App. Med. Sci., 2014; 2(6A):2000-2002.

[8]. Mehta D, Bassi R, Singh M, Mehta C. To study the knowledge about tuberculosis management and national tuberculosis program among medical students and aspiring doctors in a high tubercular endemic country. Ann Trop Med Public Health 2012; 5:206-8.

[9]. Charkazi AR, Kouchaki G, Nejad MS and Gholizade AH. Medical interns' knowledge of tuberculosis and DOTS strategy in northern Islamic Republic of Iran. EMJH 2010;16(12): 1251-1256.

[10]. Jumbo J, Ambakederemo TE and Ikuabe PO. A Survey Of Understanding Of Directly Observed Therapy Short Course (DOTS) Amongst Final Year Medical Students And Doctors. IOSR Journal of Dental and Medical Sciences (IOSR-JDMS) 2014(5): 58-62.

[11]. Mathew AS, Kakar AM. Living with tuberculosis: the myths and stigma from the Indian perspective. Clin Infect Dis 2007 Nov 1; 45(9): 1247. 